\title{
THE BRAUER GROUP OF A CURVE OVER A STRICTLY LOCAL DISCRETE VALUATION RING
}

\author{
TIMOTHY J. FORD
}

In memory of Professor S. A. Amitsur

\begin{abstract}
Aвstract. Let $K$ be the field of fractions of a curve over $R$ where $R$ is the henselization of a regular local ring on an algebraic curve over a field which is algebraically closed and has characteristic 0 . Then $K$ has the exponent $=$ degree property for division algebras. In fact every central finite dimensional $K$-division algebra with exponent $n$ is a cyclic algebra of degree $n$.
\end{abstract}

In this paper we continue to investigate the structure of division algebras $D$ finite dimensional over their center $K$. The motivating problem is to classify those fields $K$ that have the exponent $=$ degree property for division algebras. We say that $K$ has the exponent $=$ degree property if for any central $K$-division algebra $D$ the exponent of the class $[D]$ in the Brauer group $\mathrm{B}(K)$ is equal to the degree $\sqrt{(D: K)}$ of the division algebra. Throughout this paper $k$ is an algebraically closed field of characteristic 0 .

Example 1. Some fields that are known to have the exponent = degree property are listed below.

(1) A global field (an algebraic number field or a function field finitely generated of transcendence degree 1 over a finite field). This is classical.

(2) The quotient field of either (a) the henselization $\mathcal{O}_{p, X}^{h}$ or (b) the completion $\hat{\mathcal{O}}_{p, X}$ at a closed point $p$ on a normal surface $X$ over $k$ [1] or [6].

(3) The quotient field of a ring obtained by (a) henselizing or (b) completing an affine surface over $k$ along an integral curve [4].

In fact in each of these three examples, each division algebra $D$ is split by a cyclic extension $K\left(\alpha^{1 / n}\right)$ for some $\alpha \in K$ and $n=\operatorname{exponent}(D)$.

The purpose of this paper is to add to the list of Example 1 another class of fields satisfying the exponent $=$ degree property.

Let $\mathcal{O}_{p, X}$ be the local ring at a regular point $p$ on an algebraic curve $X$ over the field $k$. Then $\mathcal{O}_{p, X}$ is a local principal ideal domain, hence a discrete valuation ring. The residue field of $\mathcal{O}_{p, X}$ is $k$. Let $R=\mathcal{O}_{p, X}^{h}$ be the henselization of $\mathcal{O}_{p, X}$. Consider an affine algebraic curve $C$ over $R$. Following [9], $C$ is an affine scheme together with a structure morphism $\pi: C \rightarrow \operatorname{Spec} R$ such that $\pi$ is flat and of finite type, the fibers of $\pi$ are algebraic curves, and $C$ is connected. Then $\pi$ has 2 fibers. The closed fiber $\pi: C_{0} \rightarrow x_{0}$ over the closed point $x_{0}$ of Spec $R$ is an algebraic curve over $k$. The open fiber $\pi: C_{\eta} \rightarrow \eta$ over the open point of Spec $R$ is an algebraic curve over the quotient field of $R$. Assume that $C_{\eta}$ is integral, with

1991 Mathematics Subject Classification. 13A20, secondary 16K20, 14F20. 
$K=K\left(C_{\eta}\right)$ the field of fractions. Our main result is that $K$ has the exponent $=$ degree property for division algebras.

Theorem 2. Let $R, C$ and $K$ be as above and let $D$ be a central finite dimensional $K$ division algebra with exponent $(D)=n$. Then $D$ is a cyclic algebra of degree $n$.

Proof. The proof is in the flavor of those used by [6] and [4].

Since $C$ is $\operatorname{Spec} S$ for an algebra $S$ of finite type over $R$, we can assume $C$ is a closed subscheme of affine space $\mathbb{A}_{R}^{m}$ over $R$. Without changing $K$ we can replace $C$ with a projective completion over $R$. If necessary, we can also desingularize $C$. Therefore assume that $\pi: C \rightarrow \operatorname{Spec} R$ is proper, that the open fiber is a nonsingular integral curve $C_{\eta}$ over the quotient field of $R$. By Embedded Resolution of Curves in Surfaces [8, p. 391], we can assume that the closed fiber $\left(C_{0}\right)_{\text {red }}$ is a divisor over $k$ with normal crossings. That is, write the reduced closed fiber $\left(C_{0}\right)_{\text {red }}$ as a union $C_{1} \cup C_{2} \cup \cdots \cup C_{s}$ of irreducible curves. By the normal crossing hypothesis we assume each component $C_{j}$ is a nonsingular curve and that $\left(C_{0}\right)_{\text {red }}$ has at most ordinary double points as singularities.

Let $L / K$ be a finite extension of fields and $Y \rightarrow C$ the integral closure of $C$ in $L$. Let $f: Y^{\prime} \rightarrow Y$ be any desingularization of $Y$. That is, $Y^{\prime}$ is nonsingular and $f$ is a proper birational morphism. There is a complex

(1) $0 \rightarrow \mathrm{B}\left(Y^{\prime}\right) \rightarrow \mathrm{B}(L) \stackrel{a}{\rightarrow} \bigoplus_{\Delta} H^{1}(K(\Delta), \mathrm{Q} / \mathbb{Z}) \stackrel{r}{\rightarrow} \bigoplus_{P} \mu(-1) \stackrel{s}{\rightarrow} H^{4}\left(Y^{\prime}, \mu\right) \rightarrow 0$

which is exact except possibly at the term $\oplus H^{1}(K(\Delta), \mathbb{Q} / \mathbb{Z})$. The first summation is over all irreducible curves $\Delta \subseteq Y^{\prime}$, the second over all closed points $P \in Y^{\prime}$. This follows by combining sequences (3.1) and (3.2) of [2]. If $H^{3}\left(Y^{\prime}, \mu\right)=0$, (1) is exact. The first two groups in (1) are the Brauer groups respectively, of $Y^{\prime}$ and $L$. The map $a$ "measures the ramification" of a division algebra $\Lambda$ over $L$. The ramification divisor of $\Lambda$ is the set of divisors $\Delta$ where $a[\Lambda]$ is nontrivial. The group $H^{1}(K(\Delta), \mathbb{Q} / \mathbb{Z})$ classifies the cyclic Galois extensions of $K(\Delta)$ the function field of $\Delta$. The map $r$ measures the ramification of cyclic extensions of $K(\Delta)$. Here $\mu(-1)=\bigcup_{n} \operatorname{Hom}\left(\mu_{n}, \mathbb{Q} / \mathbb{Z}\right)$. Let $D$ be a central $K$-division algebra and $D_{L}=D \otimes L$, the restriction of $D$ to $L$. We say that $L$ splits the ramification of $D$ on $C$ if there exists a desingularization $f: Y^{\prime} \rightarrow Y$ such that the class of $D_{L}$ in the Brauer group $\mathrm{B}(L)$ is in the image of the Brauer group $\mathrm{B}\left(Y^{\prime}\right)$ of $Y^{\prime}$.

We proceed as in the proof of $[4$, Cor. 5]. Since $R$ is a direct limit of étale neighborhoods of $(X, p), C$ is of finite type over $R$ and $D$ is a finite $K$-algebra, we can find an étale neighborhood $(U, p)$ of $(X, p)$, and a nonsingular algebraic surface $C_{1}$ satisfying the following.

(1) There is a proper morphism $C_{1} \rightarrow U$.

(2) $C=C_{1} \times U$ Spec $R$

(3) If $K_{1}$ is the function field of $C_{1}$, then there is a central simple algebra $D_{1}$ over $K_{1}$ such that $D=D_{1} \otimes_{K_{1}} K$.

It was shown in the text immediately preceding Theorem 1.6 of [6] and again in [4, Prop. 3] that there exists a surface $C_{2}$ and a proper birational morphism $C_{2} \rightarrow C_{1}$ and a cyclic field extension $L_{1} / K_{1}$ of degree $n$ such that $L_{1}$ splits the ramification of $D_{1}$ on $C_{2}$. Furthermore, if $Y_{1}$ is the integral closure of $C_{2}$ in $L_{1}$, then $Y_{1}$ has only rational singularities. Let $L$ denote the field $K L_{1}$. Then $L / K$ is 
cyclic of degree $n$. Set $C^{\prime}=C_{2} \times U$ Spec $R$. Let $Y$ denote the integral closure of $C^{\prime}$ in $L$. By the construction of $C_{2}, Y$ has at most rational singularities and $D \otimes L$ is unramified at each prime divisor on $Y$. There is a desingularization $Y^{\prime} \rightarrow Y$ and $D \otimes L$ is unramified on $Y^{\prime}$. That is, $D \otimes L$ represents a class in the image of the Brauer group of $Y^{\prime}$. It therefore suffices to show that $Y^{\prime}$ has trivial Brauer group. But $Y^{\prime} \rightarrow R$ satisfies the hypothesis of Theorem 3 (which is stated and proved below), so $D \otimes L$ is split. It follows that $D$ is a cyclic algebra of degree $n$.

Theorem 3. Let $R, C$ and $K$ be as in Theorem 2. Assume moreover that $\pi: C \rightarrow \operatorname{Spec} R$ is proper, $C$ is regular, that the fibers of $\pi$ are one dimensional, and that the closed fiber of $\pi$ is a curve over $k$ with normal crossings. Then $H^{q}(C, \mu)=0$ for all $q \geq 3$ and $H^{q}\left(C, \mathbb{G}_{m}\right)=0$ for all $q \geq 2$.

Proof. Fix an integer $n \geq 2$. By proper base change $H^{q}\left(C, \mu_{n}\right) \cong H^{q}\left(C_{0}, \mu_{n}\right)$ for all $q \geq 1$, where $C_{0}$ is the closed fiber of $\pi$ (i.e. $C_{0}=C \times_{R} x_{0}$ where $x_{0}$ is the closed point of Spec $R$ ). Since $C_{0}$ is a curve over $x_{0}=\operatorname{Spec} k, H^{q}\left(C_{0}, \mu_{n}\right)=0$ for $q \geq 3$. Taking the direct limit over all $n$ gives $H^{q}\left(C_{0}, \mu\right)=0$ for $q \geq 3$. The sequence of sheaves for the étale topology on $C$

$$
\begin{aligned}
& x \longrightarrow x^{n} \\
& 1 \longrightarrow \mathrm{G}_{m} \longrightarrow \mathrm{G}_{m} \longrightarrow 1
\end{aligned}
$$

is exact by Kummer theory. The associated long exact sequence

$$
\cdots \rightarrow H^{q}\left(C, \mu_{n}\right) \rightarrow H^{q}\left(C, \mathbb{G}_{m}\right) \stackrel{n}{\rightarrow} H^{q}\left(C, \mathbb{G}_{m}\right) \rightarrow \ldots
$$

shows that multiplication by $n$ is an isomorphism on $H^{q}\left(C, \mathbb{G}_{m}\right)$ for $q \geq 3$. Since $C$ is regular, by $[7, \mathrm{II}, \mathrm{p} .71] H^{q}\left(C, \mathbb{G}_{m}\right)$ is a torsion group for all $q \geq 2$. Therefore $H^{q}\left(C, \mathbb{G}_{m}\right)=0$ for all $q \geq 3$. Now we check that the Brauer group of $C, \mathrm{~B}(C)=$ $H^{2}\left(C, \mathbb{G}_{m}\right)$, is trivial. We use the Kummer sequence (3) for $q=2$

$$
0 \rightarrow \frac{\operatorname{Pic} C}{n \text { Pic } C} \rightarrow H^{2}\left(C, \mu_{n}\right) \rightarrow{ }_{n} \mathrm{~B}(C) \rightarrow 0
$$

together with the fact that $H^{2}\left(C, \mu_{n}\right) \cong H^{2}\left(C_{0}, \mu_{n}\right)$. We assume $C_{0}$ is reduced, since $H^{2}\left(C_{0}, \mu_{n}\right) \cong H^{2}\left(\left(C_{0}\right)_{\text {red }}, \mu_{n}\right)$. Write $C_{0}=C_{1} \cup C_{2} \cup \cdots \cup C_{s}$ as a union of nonsingular irreducible curves. We assume each component $C_{j}$ is a nonsingular curve by the normal crossing hypothesis. By the Kummer sequence (4), the known description of $\mathrm{Pic}_{j} C_{j}$ and the fact that $\mathrm{B}\left(C_{j}\right)=0$ (see for example [10, pp. 175-176]), it follows that $H^{2}\left(C_{j}, \mu_{n}\right) \cong \mathbb{Z} / n$ is generated by the class of any prime divisor on $C_{j}$. Now $H^{2}\left(C_{0}, \mu_{n}\right) \cong \coprod_{j=1}^{s} H^{2}\left(C_{j}, \mu_{n}\right)$ by Lemma 4 below. It suffices to show that for each $j=1, \ldots, s$, there exists a divisor $D_{j}$ on $C$ such that $D_{j} \cap C_{j}$ is a prime divisor on $C_{j}$ and $D \cap C_{i}=\varnothing$ if $i \neq j$. Let $P_{0}$ be a prime divisor on $C_{j}$ not in the singular locus of $C_{0}$ and pick any prime divisor $D_{j}$ on $C$ such that the intersection multiplicity of $D_{j}$ and $C_{j}$ at the closed point $P_{0}$ is 1 . This is possible since $C$ and $C_{j}$ are both regular at $P_{0}$. The problem that one must worry about is the possibility that $D_{j}$ intersects $C_{0}$ at some other point. We check that this cannot happen since $D_{j}$ is integral and $R$ is henselian. Now $\iota: D_{j} \hookrightarrow C$ is a closed immersion, hence is proper. Furthermore $D_{j}$ does not contain any component $C_{i}$ of $C_{0}$. Also $D_{j}$ is closed, so $D_{j}$ does not contain $C_{\eta}$. Consider the composite $f=\pi \circ \iota: D_{j} \rightarrow \operatorname{Spec} R$. Since the fibers of $\pi$ are of dimension $1, f^{-1}(x)$ is finite 
for each $x$ in $\operatorname{Spec} R$. So $f$ is quasi-finite. Since $f$ is a composite of proper morphisms, $f$ is proper. But a proper quasi-finite morphism is finite [10, p. 6] so $D_{j}$ is finite over $R$. Since $R$ is henselian, any connected component of $D_{j} \times R x_{0}$ gives rise to a connected component of $D_{j}$. But $D_{j}$ is integral, hence $D_{j} \times_{R} x_{0}=P_{0}$. So ${ }_{n} \mathrm{~B}(\mathrm{C})=0$ for each $n>1$.

Lemma 4. Let $C$ be an algebraic curve over $k$ embedded with normal crossings on a surface $S$. If the irreducible components of $C$ are $C_{1}, \ldots, C_{s}$, then $H^{2}\left(C, \mu_{n}\right) \cong$ $\bigsqcup_{i=1}^{n} H^{2}\left(C_{i}, \mu_{n}\right)$.

Proof. Let $D=C_{1} \amalg \cdots \amalg C_{s}$ denote the disjoint union of the curves $C_{1}, \ldots, C_{s}$. There is an obvious finite projection $\pi: D \rightarrow C$. Let $\sigma C$ denote the singular locus of $C$ and $\sigma D=\pi^{-1}(\sigma C)$ those points on $D$ lying over $\sigma C$. Since $C$ has only nodal singularities, the map $\pi: \sigma D \rightarrow \sigma C$ is 2-to-1. Let $P$ be an element of $\sigma C$ and consider the cohomology with supports in $P, H_{P}^{2}\left(C, \mu_{n}\right)$. The strictly local ring $\mathcal{O}_{C, P}^{h}$ is henselian with algebraically closed residue field $k$. Let $U$ denote Spec $\mathcal{O}_{C, P}^{h}$ and let $P^{h}$ denote the closed point of $U$. By excision [10, Cor. 1.28, p. 93] $H_{P}^{2}\left(C, \mu_{n}\right) \cong H_{p h}^{2}\left(U, \mu_{n}\right)$. The long exact sequence for $P^{h} \subseteq U$ is

$$
\cdots \rightarrow H^{1}\left(U, \mu_{n}\right) \rightarrow H^{1}\left(U-P^{h}, \mu_{n}\right) \rightarrow H_{P^{h}}^{2}\left(U, \mu_{n}\right) \rightarrow H^{2}\left(U, \mu_{n}\right) \rightarrow \ldots
$$

The curve $U$ consists of 2 nonsingular henselian curves $U_{1}, U_{2}$ crossing at the closed point $P^{h}$. Each curve $U_{i}$ is the prime spectrum of a henselian discrete valuation ring with residue field $k$. So $H^{i}\left(U, \mu_{n}\right)=0$ for $i>0$ and $H^{1}(U-$ $\left.P^{h}, \mu_{n}\right)=H^{1}\left(U_{1}-P^{h}, \mu_{n}\right) \oplus H^{1}\left(U_{2}-P^{h}, \mu_{n}\right) \cong \mathbb{Z} / n \oplus \mathbb{Z} / n$. So equation (5) and excision show that $H_{P}^{2}\left(C, \mu_{n}\right) \cong(\mathbb{Z} / n)^{(2)}$. If $Q$ is an element of $\sigma D$, then the argument above also shows that $H_{Q}^{2}\left(D, \mu_{n}\right) \cong \mathbb{Z} / n$. Since $\sigma C$ decomposes into a finite number of points $P$, it follows that $H_{\sigma C}^{2}\left(C, \mu_{n}\right)$ decomposes into the direct sum $\amalg_{P \in \sigma C} H_{P}^{2}\left(C, \mu_{n}\right)$ and similarly for $\sigma D$. The long exact sequences of cohomology with supports in $\sigma C$ and $\sigma D$ combined with the maps induced by $\pi$ yield the commutative diagram below.

(6)

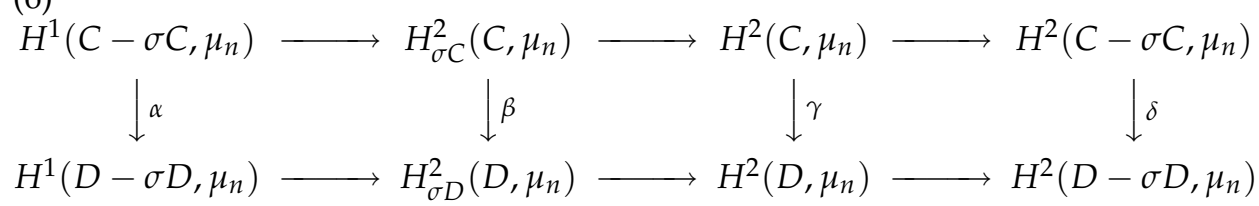

Because $\pi: C-\sigma C \stackrel{\cong}{\rightrightarrows} D-\sigma D, \alpha$ and $\delta$ are isomorphisms. The map $\beta$ is an isomorphism by the above computations. Therefore $\gamma$ is an isomorphism. Because $D$ is a disjoint union, the lemma follows.

Corollary 5. Let $R, C$ and $K$ be as in Theorem 3. The sequence

$$
0 \rightarrow \mathrm{B}(K) \stackrel{a}{\rightarrow} \bigoplus_{\Delta} H^{1}(K(\Delta), \mathrm{Q} / \mathbb{Z}) \stackrel{r}{\rightarrow} \bigoplus_{P} \mu(-1) \rightarrow 0
$$

is exact where the first summation is over all irreducible curves $\Delta \subseteq C$, the second over all closed points $P \in C$.

Proof. Follows immediately from (1) and Theorem 3. 
For any discrete valuation ring $R$ with perfect residue field $k$ and field of fractions $K$, for each $q \geq 0$ the natural map $H^{q}\left(R, \mathbb{G}_{m}\right) \rightarrow H^{q}\left(k, \mathbb{G}_{m}\right)$ is an isomorphism [7, III, p. 93]. For $q=2$ this is the theorem of Azumaya. There is a split-exact sequence

$$
0 \rightarrow H^{q}\left(k, \mathbb{G}_{m}\right) \rightarrow H^{q}\left(K, \mathbb{G}_{m}\right) \rightarrow H^{q-1}\left(k, \mathbb{G}_{m}\right) \rightarrow 0
$$

for each $q \geq 2$ [7, III, p. 93 and p. 188]. In particular, for $q=2$, it follows that the Brauer group of $K$ decomposes into a direct sum of $\mathrm{B}(k)$ and $H^{1}(k, \mathbb{Q} / \mathbb{Z})$. The group $H^{1}(k, \mathbb{Q} / \mathbb{Z})$ parametrizes the unramified cyclic Galois extensions of $K$. Various other results along these same lines are derived in [3] and [11]. We arrive at similar results for the Brauer group of a curve over $K$.

Theorem 6. Let $R$ be a strictly local ring which is the henselization of a local ring $\mathcal{O}_{p, X}$ at a closed point $p$ on a smooth curve X over $k$. Let $\pi: C \rightarrow \operatorname{Spec} R$ be proper and smooth of relative dimension 1 . Let $\eta$ be the generic point of $\operatorname{Spec} R$ and $C_{\eta}=C \times_{R} \eta$. Let $x_{0}$ be the closed point of Spec $R$ and $C_{0}=C \times_{R} x_{0}$. Then the Brauer group $\mathrm{B}\left(C_{\eta}\right)$ is isomorphic to $H^{1}\left(C_{0}, Q / Z\right)$. Every Azumaya algebra over $C_{\eta}$ of exponent $n$ is split by a cyclic Galois extension of degree $n$ which descends to an unramified extension of $C$.

Proof. Since Spec $R=\left\{x_{0}\right\} \cup\{\eta\}$ with $\left\{x_{0}\right\}$ closed and $\{\eta\}$ open, we have $C=$ $C_{0} \cup C_{\eta}$ with $C_{0}$ closed and $C_{\eta}$ open. The long exact sequence of cohomology with supports in $C_{0}$ and coefficients in $\mathbb{G}_{m}$ is

(7) $H^{0}\left(C, \mathbb{G}_{m}\right) \rightarrow H^{0}\left(C_{\eta}, \mathbb{G}_{m}\right) \rightarrow H_{C_{0}}^{1}\left(C, \mathbb{G}_{m}\right) \rightarrow$

$$
\begin{aligned}
H^{1}\left(C, \mathbb{G}_{m}\right) \rightarrow & H^{1}\left(C_{\eta}, \mathbb{G}_{m}\right) \rightarrow H_{C_{0}}^{2}\left(C, \mathbb{G}_{m}\right) \rightarrow \\
& H^{2}\left(C, \mathbb{G}_{m}\right) \rightarrow H^{2}\left(C_{\eta}, \mathbb{G}_{m}\right) \rightarrow H_{C_{0}}^{3}\left(C, \mathbb{G}_{m}\right) \rightarrow \ldots
\end{aligned}
$$

If $t$ is a local parameter for $R$, then $\frac{H^{0}\left(C_{\eta}, \mathbb{G}_{m}\right)}{H^{0}\left(C, G_{m}\right)}=\langle t\rangle$ and $t$ vanishes with order 1 along $C_{0}$. Since $C_{0}$ is a principal divisor, $\operatorname{Pic} C \cong \operatorname{Pic} C_{\eta}$. From Kummer theory the diagram

(8)

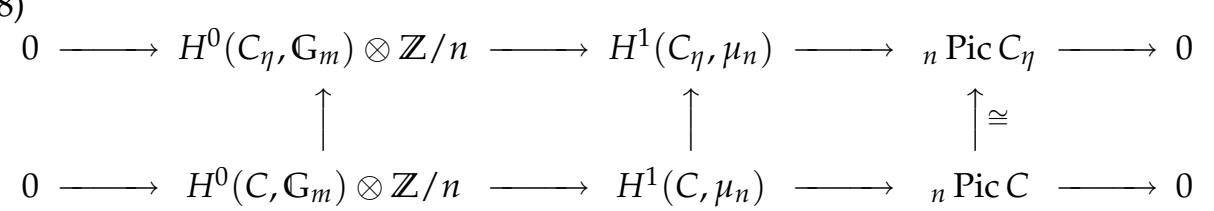

commutes and has exact rows. Since $H^{0}\left(C_{\eta}, \mathbb{G}_{m}\right) \otimes \mathbb{Z} / n \cong\langle t\rangle /\left\langle t^{n}\right\rangle$ and $H^{0}\left(C, \mathbb{G}_{m}\right) \otimes$ $\mathbb{Z} / n=0$, we see from (8) that $H^{1}\left(C_{\eta}, \mu_{n}\right) \cong\langle t\rangle /\left\langle t^{n}\right\rangle \times H^{1}\left(C, \mu_{n}\right)$ and by proper base change $H^{1}\left(C, \mu_{n}\right) \cong H^{1}\left(C_{0}, \mu_{n}\right)$. From Theorem 3 we have $H^{q}\left(C, \mathbb{G}_{m}\right)=0$ for all $q \geq 2$. From $(7)$ it follows that $H^{q}\left(C_{\eta}, \mathbb{G}_{m}\right) \cong H_{C_{0}}^{q+1}\left(C, \mathbb{G}_{m}\right)$ for all $q \geq 2$. Since Spec $R$ is a direct limit of étale neighborhoods $U \hookrightarrow X$ of the closed point $p \hookrightarrow X$, the morphism $\pi: C \rightarrow \operatorname{Spec} R$ descends to a proper smooth morphism $C^{\prime} \rightarrow U$ with a closed fiber $C_{0}^{\prime}=C^{\prime} \times_{U} p \cong C_{0}$. By cohomological purity (see [5]) $H_{C_{0}^{\prime}}^{q}\left(C^{\prime}, G_{m}\right) \cong H^{q-2}\left(C_{0}, \mathbb{Q} / \mathbb{Z}\right)$ for all $q \geq 3$. Taking the limit over all such $U \hookrightarrow X$ yields $H_{C_{0}}^{q}\left(C, G_{m}\right) \cong H^{q-2}\left(C_{0}, \mathbb{Q} / \mathbb{Z}\right)$ for all $q \geq 3$. Therefore $H^{2}\left(C_{\eta}, \mathbb{G}_{m}\right) \cong H^{1}\left(C_{0}, \mathbb{Q} / \mathbb{Z}\right), H^{3}\left(C_{\eta}, \mathbb{G}_{m}\right) \cong H^{2}\left(C_{0}, \mathbf{Q} / \mathbb{Z}\right) \cong \mu$ and $H^{q}\left(C_{\eta}, \mathbb{G}_{m}\right) \cong$ $H^{q-1}\left(C_{0}, \mathbb{Q} / \mathbb{Z}\right)=0$ for all $q \geq 4$. The isomorphism ${ }_{n} H^{2}\left(C_{\eta}, \mathbb{G}_{m}\right) \cong H^{1}\left(C_{0}, \mathbb{Z} / n\right)$ 
is the Gysin map [10, p. 244]. Given any $\lambda$ in $H^{1}(C, \mathbb{Z} / n)$ there is a corresponding $\lambda_{0}$ in $H^{1}\left(C_{0}, \mathbb{Z} / n\right)$. Let $\lambda_{\eta}$ denote the image of $\lambda$ in the group $H^{1}\left(C_{\eta}, \mathbb{Z} / n\right)$ under the natural map. Let $\lambda_{\eta}$ also denote the corresponding cyclic Galois cover of $C_{\eta}$ with group $\langle\sigma\rangle$. Using the cyclic Galois cover $\lambda_{\eta}$ and the trivial factor set $t$ we form a cyclic crossed product algebra $\Delta(\lambda)=\left(\lambda_{\eta} / C_{\eta}, \sigma, t\right)$ which represents a class in ${ }_{n} \mathrm{~B}\left(C_{\eta}\right)$. Consider the ramification divisor of $\Delta(\lambda)$ on $C$. Along the divisor $C_{0}$ of $C$, the ramification of $\Delta(\lambda)$ is the element $\lambda_{0}$ of the group $H^{1}\left(C_{0}, \mathbb{Z} / n\right)$. Therefore, the correspondence $\lambda \mapsto \Delta(\lambda)$ induces an isomorphism $H^{1}(C, \mathbb{Z} / n) \cong{ }_{n} \mathrm{~B}\left(C_{\eta}\right)$. Every Azumaya algebra over $C_{\eta}$ whose Brauer class is annihilated by $n$ is Brauer equivalent to a cyclic crossed product of the form $\left(\lambda_{\eta} / C_{\eta}, \sigma, t\right)$, hence is split by $\lambda_{\eta}$ for some $\lambda$.

\section{REFERENCES}

[1] M. Artin, Two dimensional orders of finite representation type, Manuscripta Math., 58 (1987), pp. 445471.

[2] M. Artin and D. Mumford, Some elementary examples of unirational varieties which are not rational, Proc. London Math. Soc. (3), 25 (1972), pp. 75-95.

[3] M. Auslander and A. Brumer, Brauer groups of discrete valuation rings, Nederl. Akad. Wetensch. Proc. Ser. A, 71 (1968), pp. 286-296.

[4] T. J. Ford, Division algebras over nonlocal henselian surfaces, Pacific J. Math., 147 (1991), pp. 301-310.

[5] - On the Brauer group of a localization, J. Algebra, 147 (1992), pp. 365-378.

[6] T. J. Ford and D. Saltman, Division algebras over henselian surfaces, Israel Mathematical Conference Proceedings, 1 (1989), pp. 320-336.

[7] A. Grothendieck, Le groupe de Brauer I, II, III, in Dix Exposés sur la Cohomologie des Schémas, North Holland, Amsterdam, 1968, pp. 46-188.

[8] R. Hartshorne, Algebraic Geometry, vol. 52 of Graduate Texts in Mathematics, Springer-Verlag, New York/Berlin, 1977.

[9] S. Lichtenbaum, Curves over discrete valuation rings, Amer. J. Math., 90 (1968), pp. 380-405.

[10] J. Milne, Etale Cohomology, vol. 33 of Princeton Mathematical Series, Princeton University Press, Princeton, N.J., 1980.

[11] S. Yuan, On the Brauer groups of local fields, Ann. of Math. (2), 82 (1965), pp. 434-444.

Department of Mathematics, Florida Atlantic University, Boca Raton, FL 33431

E-mail address: Ford@acc.fau.edu 